\title{
La epidemia de obesidad y sus factores relacionados: el caso de España
}

\author{
The obesity epidemic and related \\ factors: the case of Spain
}

Juan Luis Gutiérrez-Fisac 1

Enrique Regidor 2

Esther López García 1

Jose Ramón Banegas Banegas 1

Fernando Rodríguez Artalejo 1

\footnotetext{
1 Departamento de Medicina Preventiva y Salud Pública, Facultad de Medicina, Universidad Autónoma de Madrid. Arzobispo Morcillo s/n, 28029 Madrid, España. jgutierrez@msc.es 2 Departamento de Medicina Preventiva y Salud Pública Facultad de Medicina, Universidad Complutense de Madrid. Ciudad Universitaria s/n, 28040 Madrid, España.
}

Abstract The obesity epidemic is a global phenomenon that does not respect geographic or socio-demographic boundaries. Thus, research on factors related to the obesity epidemic has focused on social and economic characteristics of modern societies. This article discusses obesity in Spain and trends in several associated factors. Together with the increase in the prevalence of obesity, important changes in the population's dietary pattern have been observed, although total energy and fat intake appear to be stable. According to several indirect indicators, sedentary behavior predominates, although the percentage of the population reporting someleisure exercise is increasing. An increase in the amount of lei sure time in modern soci eties is suggested as an explanation for this paradox. Factors related to energy expenditure could be as important as di etary factors in the genesis of the obesi ty epi demic.

Key words Obesity; Diet; Feeding Behavior

Resumen La epidemia de obesidad es un fenómeno universal que no parece reconocer límites ni geográficos ni sociodemográficos. Las investi gaci ones sobre los factores responsables de la epidemi a se centran sobre algunas de las condi ci ones sociales y económi cas que imperan en las sociedades actuales. El presente artículo muestra la situación de la obesidad en España y la tendencia de una serie de factores potencialmente relacionados. El incremento en Ia prevalencia de obesi dad se acompañó de importantes cambios en el patrón de alimentación de la población a pesar de una tendencia estabilizada en la ingesta calórica total y de grasas. Diversos indicadores indirectos muestran la tendencia sedentaria de la población, a pesar de que un mayor porcentaje de la población declara realizar ejercicio físico en su tiempo libre. Los factores dependientes del gasto energéti co parecen tan o más importantes que los derivados del ingreso. Para explicar la si tuación paradóji ca de los distintos indicadores de sedentarismo se sugi ere la hi pótesi s de un aumento del "stock de ti empo libre" en las soci edades actuales.

Palabras clave Obesidad; Di eta; Conducta Alimentaria 


\section{Introducción}

Pocos asuntos en el campo de la salud pública han tenido en los últimos años tanta repercusión en los medios de comunicación científicos como la epidemia de obesidad. Las revistas biomédicas muestran con frecuencia editoriales, trabajos de revisión y opinión y suplementos especiales que ponen de manifiesto el continuo y, en algunos casos, dramático incremento en la frecuencia de la obesidad en la población (Hill \& Peters, 1998; Khan \& Bowman, 1999; Pi-Sunyer, 1994; Prentice \& Jebb, 1995). Si bien al inicio de la década de los ochenta este incremento en la prevalencia de obesidad era observado exclusivamente en los países económicamente desarrollados (Seidell, 1995), a medida que se va disponiendo de información en países con menor nivel de desarrollo, se comprueba que la epidemia de obesidad es un fenómeno universal. Así, países como Brasil, Chile, Mauritania, China o India muestran incrementos en la prevalencia de obesidad en sus poblaciones (Popkin \& Doak, 1998; Uauy et al., 2001).

Aunque existen importantes diferencias en la magnitud del aumento de la prevalencia de obesidad entre unos grupos de población y otros, siendo bien conocido el mayor incremento relativo en los individuos con un menor nivel socioeconómico o en las edades medias de la vida (Flegal et al., 1988a, 1988b; Galuska et al., 1996; Gutiérrez-Fisac et al., 2000; Seidell et al., 1995), la obesidad afecta a todos los grupos sociodemográficos. Esta enorme generalización de la epidemia, que no parece reconocer límites ni geográficos ni sociodemográficos, ha hecho que las investigaciones sobre los factores poblacionales responsables de tan Ilamativos cambios se centren sobre algunas de las condiciones sociales y económicas que imperan en las sociedades actuales.

Numerosos trabajos han sugerido que ciertos factores relacionados tanto con la alimentación como con la actividad física serían los responsables de la tendencia creciente en la frecuencia de la obesidad (Fogelholm et al., 1996; Hill \& Melanson, 1999; Hill \& Peters, 1998; Prentice \& J ebb, 1995; Stamler, 1993). En cuanto a la dieta, se ha mencionado que cierto incremento en la ingesta energética vendría dado por la alta disponibilidad de alimentos baratos de al to contenido energético, o por el incremento en el tamaño de las raciones, ligado al hábito cada vez más frecuente de comer fuera de casa. Con respecto al ejercicio físico, una tendencia al comportamiento sedentario promovido por la mecanización creciente tanto de los medios de transporte como de las ac- tividades del tiempo libre o del trabajo, serían los principales factores asociados a un menor gasto de energía. Sin embargo, excepto algunos trabajos (Fogelholm et al., 1996; Prentice \& Jebb, 1995), o estudios puntuales sobre alguno de los aspectos mencionados (French et al., 2000; Jeffery \& French, 1998; Rodríguez-Artalejo et al., 2002), la mayoría de estos trabajos no muestran información sobre la situación de los factores propuestos como responsables del incremento de la prevalencia de la obesidad, ni sugieren hipótesis sobre cuáles de ellos son los más importantes o a través de qué mecanismos actúan.

El objetivo del presente trabajo es mostrar el incremento en la prevalencia de la obesidad en la población adulta española, su distribución entre los principales grupos sociodemográficos y presentar la tendencia de aquellos factores potencialmente responsables de los que se dispone de información. De esta forma se sugieren hipótesis sobre los principales factores implicados en la epidemia de obesidad.

\section{Material y métodos}

El presente trabajo utiliza diversas fuentes de información. Los datos sobre la prevalencia y la distribución de la obesidad y el sedentarismo en el tiempo libre proceden de las encuestas nacionales de salud que desde 1987 se han realizado periódicamente en España, tal y como fueron publicados en I ndicadores de Salud (Regidor \& Gutiérrez-Fisac, 1999). Se muestran únicamente datos para 1987 y 1997 con el objeto de simplificar la presentación y a la vez examinar el periodo temporal más amplio posible. Las características de dichas encuestas han sido detalladas en otro lugar (Gutiérrez-Fisac et al., 2000). En resumen, tanto en 1987 como en 1997, se seleccionó una muestra representativa de la población española no institucionalizada mediante un muestreo estratificado y en diferentes etapas en el que las unidades de primera etapa (municipios) y las de segunda (distritos censales) fueron seleccionadas por muestreo aleatorio simple, y las unidades de tercera etapa (individuos) mediante cuotas de edad y sexo. Se realizaron 29.647 y 6.400 entrevistas a individuos de 16 y más años en 1987 y 1997 respectivamente.

La obesidad se estimó por el índice de masa corporal (IMC), definido como el peso, en kilogramos, dividido por la talla en metros al cuadrado. El peso y la talla fueron respondidos personalmente por el entrevistado a través de las siguientes dos preguntas incluidas en los 
cuestionarios: ¿Podría decirme cuánto pesa usted, aproximadamente, sin zapatos ni ropa?Y ¿podría decirme cuánto mide sin zapatos? A pesar de que existe una tendencia a sobrestimar la talla e infraestimar el peso, se ha comprobado que la declaración del peso y la talla por parte del individuo tiene una sensibilidad y una especificidad aceptables (Nieto-García et al., 1990). Se consideraron obesos a los individuos con un IMC $\geq 30 \mathrm{~kg} / \mathrm{m}^{2}$ (NIH, 1998).

La actividad física en el tiempo libre se obtuvo de la siguiente pregunta: ¿Cuál de las siguientes posibilidades describe mejor su actividad en su tiempo libre?: (1) Casi completamente inactivo (leer, ver la televisión, ir al cine, etc.); (2) Alguna actividad física o deportiva ocasional o esporádica (caminar o pasear en bicicleta, jardinería, gimnasia suave, actividades recreativas de ligero esfuerzo, etc.); (3) Actividad física regular varias veces al mes (tenis, gimnasia, correr, natación, ciclismo, juegos de equipo, etc.) y (4) Entrenamiento físico varias veces por semana. Se consideraron sedentarios los individuos que contestaron la opción 1.

Las prevalencias de obesidad para la población de 20 y más años se presentan separadamente para hombres y mujeres, desagregadas por grupos de edad y por nivel de estudios. Las prevalencias por esta última variable fueron ajustadas por edad siguiendo el método directo y utilizando como población estándar la de 1987. Se obtuvieron, asimismo, los porcentajes de cambio entre uno y otro año. La prevalencia de sedentarismo se presenta en hombres y mujeres de 16 y más años.

Por otra parte, se estudian dos indicadores de la tendencia, entre 1980 y 1997, en la dieta de la población española: la ingesta media de energía por persona y día (en kilocalorías) y el porcentaje de la ingesta total debida a grasas. Los datos, que corresponden a disponibilidad alimentaria, fueron obtenidos de la base de datos Salud para Todos, de la Organización Mundial de la Salud (WHO, 2000).

Finalmente, se estudió la tendencia temporal, entre 1987 y 1997, de diversos indicadores indirectos de sedentarismo: el número de vehículos turismos en funcionamiento por 10.000 habitantes, obtenido de los anuarios estadísticos del Instituto Nacional de Estadística (INE, 1994a, 2001); el promedio de minutos de visionado de televisión por persona y día y el porcentaje de hogares con distintos equipamientos (dos o más televisores, mando a distancia deT.V., aparato de vídeo, ordenador personal y dos o más coches, compact disc, microondas, secadora de ropa, lavavajillas y aspiradora), obtenidos del Estudio General de Medios (AIMC, 2001).

\section{Resultados}

Las Tablas 1 y 2 muestran la prevalencia de obesidad en 1987 y 1997 en los hombres y mujeres españolas de 20 y más años, así como el cambio absoluto en el periodo estudiado. Como puede verse, la obesidad aumentó en un 5\% entre uno y otro año, lo que supuso un cambio relativo del $71 \%$ en los hombres y del $63 \%$ en las mujeres.

Por edad, se observaron incrementos en todos los grupos. En los hombres (Tabla 1) fueron las edades medias en las que la prevalencia aumentó más. Así, en los grupos de 35 a 44, 45 a 54 y 55 a 64 años, el incremento fue del 6, 6,3 y $8,1 \%$ respectivamente. Entre las mujeres ( $\mathrm{Ta}$ bla 2) los mayores incrementos se observaron a partir de los 45 años. En la mayoría de los grupos de edad, el aumento de la prevalencia de obesidad fue mayor en los hombres que en las mujeres.

Según el nivel de estudios, las prevalencias fueron mayores, tanto en uno como en otro se$x o$, en los individuos sin estudios (Tablas 1 y 2). Entre los hombres se produjo un incremento en todos los niveles de estudios, si bien el cambio absoluto en la prevalencia fue mayor en los individuos sin estudios, en los que creció un $12,6 \%$. Este incremento fue del 7,2, 6,3 y 5,7\% en los sujetos de estudios primarios, secundarios y terciarios respectivamente.

Tabla 1

Prevalencia de obesidad (IMC $\geq 30 \mathrm{~kg} / \mathrm{m}^{2}$ ) y cambio absoluto de la obesidad entre 1987 y 1997 en hombres españoles de 20 y más años.

\begin{tabular}{lrrc}
\hline & 1987 & 1997 & Cambio absoluto* \\
\hline Edad & & & \\
$20-24$ & 1,6 & 3,5 & 1,9 \\
$25-34$ & 4,4 & 7,5 & 3,1 \\
$35-44$ & 7,6 & 13,6 & 6,0 \\
$45-54$ & 9,3 & 15,6 & 6,3 \\
$55-64$ & 11,2 & 19,3 & 8,1 \\
65 y más & 10,4 & 15,4 & 5,0 \\
& & & \\
Nivel de estudios** & & & 12,6 \\
Sin estudios & 9,2 & 21,8 & 7,2 \\
Estudios primarios & 8,5 & 15,7 & 6,3 \\
Estudios secundarios & 5,2 & 11,5 & 5,7 \\
Estudios terciarios & 4,4 & 10,1 & 5,1 \\
& & & \\
Total & 7,2 & 12,3 & \\
\end{tabular}

* Cambio absoluto = prevalencia 1997 - prevalencia 1987

** Prevalencias ajustadas por edad. 
Tabla 2

Prevalencia de obesidad (IMC $\geq 30 \mathrm{~kg} / \mathrm{m}^{2}$ ) y cambio absoluto de la obesidad entre 1987 y 1997 en mujeres españolas de 20 y más años.

\begin{tabular}{lrrr}
\hline & 1987 & 1997 & Cambio absoluto* \\
\hline Edad & 0,4 & 0,7 & 0,3 \\
$20-24$ & 3,0 & 6,2 & 3,2 \\
$25-34$ & 6,3 & 8,0 & 1,7 \\
$35-44$ & 13,7 & 17,9 & 4,2 \\
$45-54$ & 17,1 & 24,1 & 7,0 \\
$55-64$ & 16,3 & 25,4 & 9,1 \\
65 y más & & & \\
& 13,5 & 20,2 & 6,7 \\
Nivel de estudios** & 8,2 & 15,8 & 7,6 \\
Sin estudios & 3,8 & 11,2 & 7,4 \\
Estudios primarios & 3,9 & 2,6 & $-1,3$ \\
Estudios secundarios & & & 5,2 \\
Estudios terciarios & 8,3 & 13,5 & \\
\hline Total & & &
\end{tabular}

* Cambio absoluto = prevalencia 1997 - prevalencia 1987

** Prevalencias ajustadas por edad.

En las mujeres, la prevalencia de obesidad fue también mayor entre las de menor nivel de estudios, y aumentó entre 1987 y 1997 en todos los grupos excepto en las de estudios terciarios, en las que la prevalencia descendió un 1,3\%.

La Figura 1 muestra la tendencia desde el inicio de la década de los años ochenta de la disponibilidad media de kilocalorías por persona y día y el porcentaje de la misma debida a grasas. El número medio de kilocalorías por persona y día pasó de 3.061 en 1980 a 3.297 en 1997, si bien desde el inicio de la década de los años noventa está estabilizado. El porcentaje de la energía debida a las grasas también aumentó desde 1980 (32,9\%) a 1997 (39,6\%), y aunque se observa un continuado incremento, dicho aumento en los últimos años es muy pequeño.

En la Figura 2 pueden verse las prevalencias de sedentarismo en el tiempo libre en la población adulta española entre 1987 y 1997. Tanto en uno como en otro sexo, el porcentaje de población que no realiza ejercicio físico alguno durante su tiempo libre disminuyó entre 1987 y 1997, pasando del 70,7 al 51,8\% y del 53,7 al $39,1 \%$ en mujeres y hombres respectivamente.

El número de vehículos por 10.000 habitantes creció ininterrumpidamente, pasando de ser 2.640 por 10.000 habitante en 1987 a 3.890 en 1997 (Figura 3). Esa misma figura muestra un ligero incremento en el número medio de minutos de visionado de televisión por perso- na y día en el periodo de estudio, promedio que era de 209 minutos en 1987 y que pasó a ser de 231 en 1997.

Finalmente, la Tabla 3 presenta el porcentaje de hogares españoles con diferentes equipamientos en 1988 y 1997. Como puede apreciarse se produjo un notable aumento en todos los indicadores seleccionados. El porcentaje de hogares con dos o más aparatos de televisión pasó del 21,6 al 57,8\% entre esos dos años, lo que supuso un porcentaje de cambio del $168 \%$. Un incremento similar en términos relativos se produjo en el porcentaje de hogares con dos o más coches (196\%) o con lavavajillas (200\%). Los porcentajes de hogares con ordenador personal y mando a distancia de TV pasaron del 5,1 al 21,9\% (porcentaje de cambio del 329\%) y del 12,8 al $80 \%$ (porcentaje de cambio del 525\%) respectivamente entre 1988 y 1997.

\section{Discusión}

Los datos presentados muestran un aumento en la prevalencia de obesidad de la población adulta española del 5\% entre los años 1987 y 1997, Io que supuso un crecimiento relativo superior al $60 \%$ en esos diez años. Este aumento en el porcentaje de población que podría ser considerado obesa es similar al observado en otros países. En Estados Unidos, durante la década de los ochenta y principios de los noventa, se observaron incrementos anuales medios de la prevalencia de obesidad del 0,5-1\% (Galuska et al., 1996). Incrementos similares a este se produjeron durante la década de los años 80 en Australia y Canadá (Seidell, 1995). En Europa se observaron tendencias crecientes en la prevalencia de obesidad en la mayoría de los países con información disponible: en Inglaterra, la prevalencia de obesidad se dobló entre 1980 y 1993 (Department of Health, 1995); en Holanda (Seidell, 1995), Alemania (H offmeister et al., 1994), Suecia (Kuskowska-Wolk \& Bergström, 1993a, 1993b) o Francia (Maillard et al., 1999) se observó igualmente una tendencia creciente. En términos relativos, entre el final de la década de los años 80 y mitad de los 90, la obesidad aumentó en la mayoría de estos países entre un 10 y un $40 \%$ (Björntorp, 1997). Dicho incremento no es exclusivo del mundo económicamente desarrollado, como inicialmente se pensó, sino que afecta a otros países con menor desarrollo socioeconómico. La revisión de Popkin sobre datos epidemiológicos a nivel mundial muestra que en países como Brasil, India, China o Mauritania, se han venido produciendo incrementos en los porcentajes de 


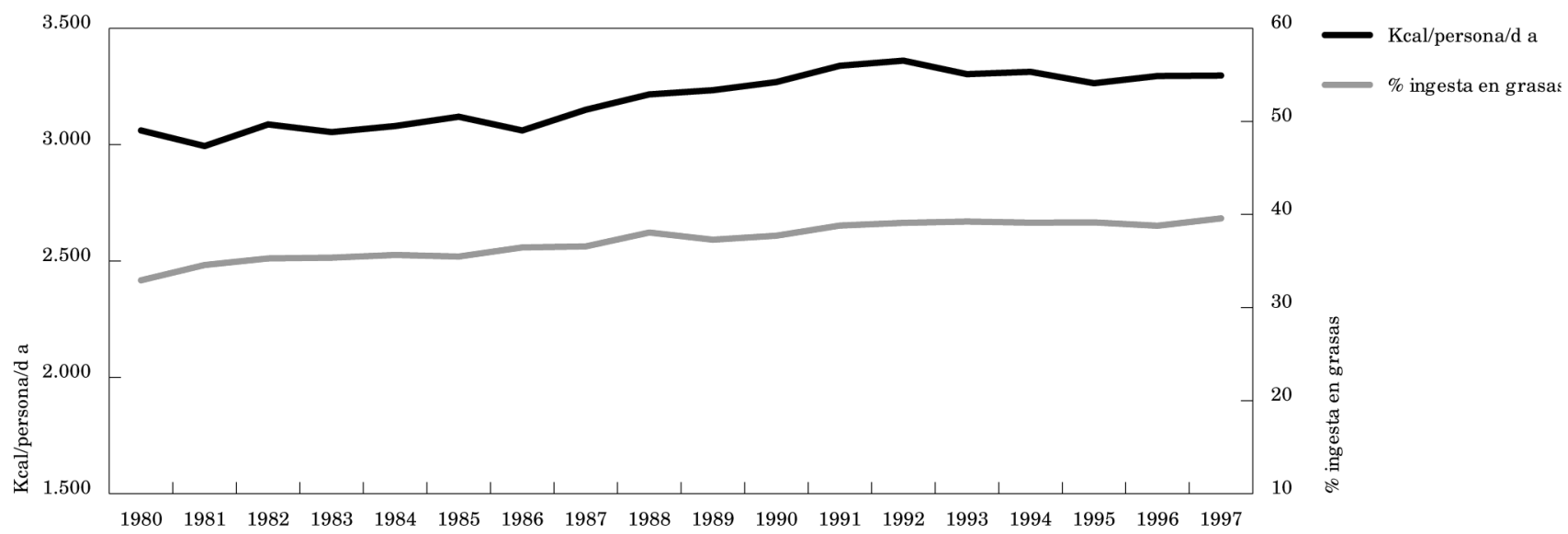

población con sobrepeso y obesidad (Popkin \& Doak, 1998).

Los datos mostrados señalan también que el incremento en la prevalencia de la obesidad afecta a todos los grupos sociodemográficos. Aunque existen interesantes variaciones en la magnitud del cambio en la frecuencia de la obesidad entre unos grupos y otros, no es el objetivo del presente trabajo examinar dichos cambios, sino constatar que, como ocurre en otros países, los factores que se esconden detrás de la epidemia de obesidad afectan de forma indiscriminada a todos los estratos de la población. Este hecho, junto a la ya mencionada extensión de la epidemia a países de muy diferente desarrollo socioeconómico, sugiere que los factores responsables de la tendencia creciente en la prevalencia de la obesidad están íntimamente ligados al desarrollo socioeconómico.

Multitud de revisiones han tratado de analizar los factores implicados en la tendencia creciente de la obesidad. En muchos de ellos se establecen dos argumentos que sirven de punto de partida para el análisis posterior, y que los autores del presente trabajo suscriben. Por una parte, la escasa rel evancia de los factores genéticos. Si bien la influencia de dichos factores en algunos tipos de obesidad es innegable, como lo es el poder explicativo de la dotación genética a la hora de establecer diferencias interindividuales en la capacidad de acumular grasa a igual ingesta y gasto, los factores gené-
Figura 2

Prevalencia de sedentarismo en el tiempo libre en 1987 y 1997, en hombres y mujeres españolas de 16 y más años.

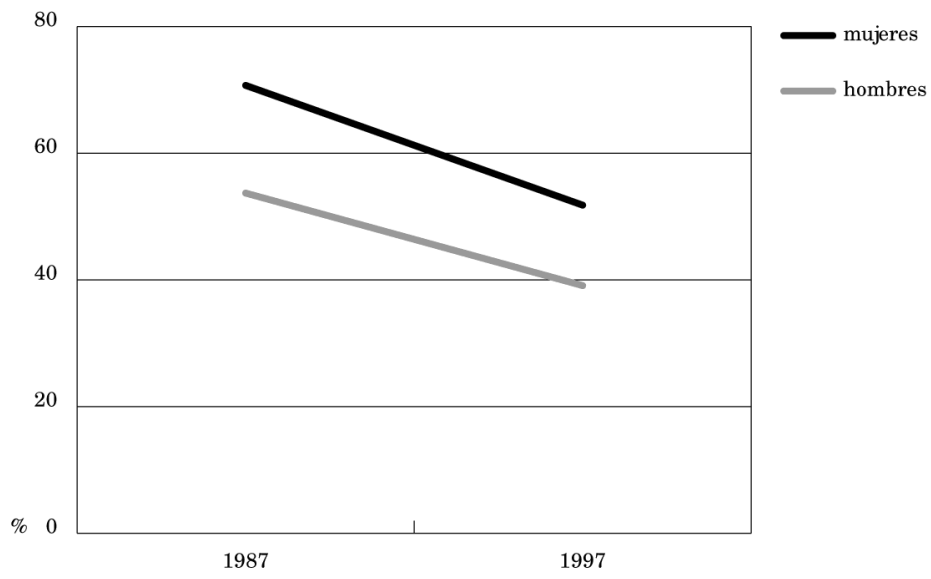


Figura 3

Vehículos turismos (en miles) en circulación por 10.000 habitantes y minutos promedio de visionado

de televisión por persona y día, 1987-1997.

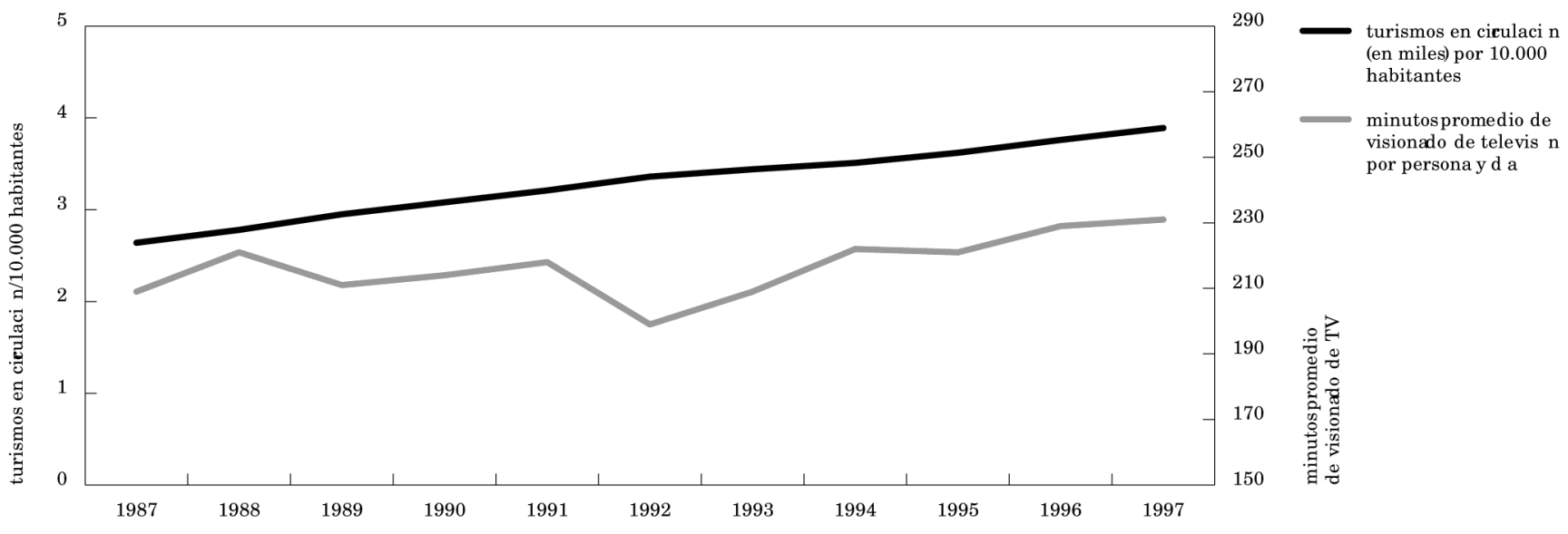

ticos no tendrían influencia alguna en la tendencia creciente de la obesidad, dada la extraordinaria rapidez con la que se ha producido. De esta forma, el debate sobre las causas implicadas se centra en los factores medioambientales.

Por otra parte, a la hora de estudiar los factores responsables de la epidemia, se asume que, igual que ocurre a nivel individual, dichos factores estarán influyendo en el balance energético. Por lo tanto, la cuestión se reduce a examinar los cambios durante las décadas pasadas en la dieta y el patrón de alimentación de las poblaciones y en el patrón dominante de actividad física de las mismas.

El análisis de los patrones dietéticos de las poblaciones suele hacerse en base a la información que procede de hojas de balance alimentario y encuestas a los hogares de presupuestos familiares o de encuestas de nutrición. No existen, para el conjunto de España, estimaciones poblacionales basadas en la frecuencia de consumo de alimentos o en recordatorios de 24 horas, instrumentos básicamente utilizados en las encuestas de nutrición, por lo que los datos disponibles proceden de hojas de balance alimentario y de encuestas de presupuestos familiares. Si bien hay constancia de que la disponibilidad media de energía de la población española vino aumentando desde los años cincuenta (Rodríguez-Artalejo et al., 1996), también es cierto que en los últimos quince años la tendencia está estabilizada. De igual forma, el porcentaje de la energía disponible en forma de grasas, otro de los indicadores dietéticos comúnmente empleados, ha seguido una evolución parecida, aunque todavía se observa un ligero incremento. La prevalencia de obesidad ha aumentado en los últimos años sin que lo haya hecho, de forma sustancial, la ingesta de calorías y de grasas.

Esta misma tendencia paradójica entre los datos de obesidad y los de ingesta se ha visto en Inglaterra (Prentice \& Jebb, 1995), Finlandia (Folgelholm et al., 1996) o Estados Unidos (Heini \& Weinsier, 1997), con información sobre consumo de alimentos obtenida mediante distintos instrumentos en encuestas a muestras representativas de la población. Por tanto, esta situación que dibujan los datos sobre la influencia de la dieta en la obesidad podría ser real, y entonces las hipótesis que proponen al descenso en el gasto de energía como el principal factor implicado en la epidemia de obesidad serían correctas. Pero también la paradoja entre unos datos y otros podría ser debida a la mala calidad de la información sobre la dieta de las poblaciones. Es posible que ninguno de los instrumentos de medición de la dieta de las poblaciones, directos o indirectos, refleje con exactitud la ingesta de energía. Sin embargo, esta falta de exactitud no tendría por qué verse afectada a lo largo del tiempo, y las tendencias podrían estar reflejando con validez los cambios en la disponibilidad de energía. Otra razón, menos estudiada, podría ser que dichos 
instrumentos de medición de la dieta de las poblaciones no sean sensibles para captar ciertos cambios importantes que podrían estar produciéndose en el patrón de alimentación de las sociedades actuales. Algunos autores han mencionado que la industria alimentaria ha trasformado muchos de los alimentos más consumidos. Se ha descrito, por ejemplo, que algunos de los nuevos alimentos bajos en grasas tendrían una densidad calórica tan alta o mayor que la de los alimentos a los que reemplazan (Hill \& Peters, 1998).

Otros factores que han sido mencionados y que podrían igualmente quedar fuera del alcance de los instrumentos de medición indirecta de la dieta de las poblaciones son el consumo de alimentos fuera del hogar y, en íntima relación con ello, el consumo de lo que se ha denominado la comida rápida. Ya hay al guna evidencia epidemiológica de que las comidas fuera del hogar podrían estar contribuyendo al aumento de la prevalencia de la obesidad (Binkley et al., 2000) y de que el consumo de este tipo de alimentos se asocia a una mayor ingesta de energía y de grasas y a un mayor peso corporal (French et al., 2000; McCrory et al., 1999). Además, es precisamente en el seno de la oferta de los restaurantes donde podría haberse producido, más que en los consumos de alimentos en el hogar, un incremento en el tamaño de las raciones (French et al., 2001), otro de los factores comúnmente mencionados ( Hill \& Melanson, 1999).

La escasa información disponible en España sobre estos nuevos factores del comportamiento alimentario muestra que se ha producido un aumento tanto del gasto medio por persona y año en alimentos consumidos fuera del hogar, como en el porcentaje de gasto en alimentación fuera del hogar, que pasó de un $16 \%$ a un $24 \%$ entre 1981 y 1991 . I gualmente se produjo una reducción en el número de comidas y cenas realizadas en el hogar durante el mismo periodo (INE, 1994b). En países como Estados Unidos, donde se dispone de mucha información sobre estos factores, se ha comprobado que entre los años 70 y los años 90 se produjo un importante aumento en el número de restaurantes de comida rápida, en el porcentaje de comidas realizadas fuera del hogar y en el gasto en comidas fuera del hogar, que pasó de un $25 \%$ del total del gasto en alimentación en 1970 a un 53\% en 1995 (French et al., 2001).

Finalmente, habría que contemplar la posibilidad de que las actuales prevalencias de sobrepeso y obesidad sean el resultado de un efecto acumulativo por la creciente disponibilidad
Tabla 3

Porcentaje de hogares españoles con diferentes equipamientos. 1987 y 1997.

\begin{tabular}{lrcc}
\hline & 1988 & 1997 & \% de cambio 1 \\
\hline Dos o más televisores & 21,6 & 57,8 & 168 \\
Mando a distancia de TV & 12,8 & 80,0 & 525 \\
Aparato de vídeo & 25,9 & 66,9 & 158 \\
Ordenador personal & 5,1 & 21,9 & 329 \\
Dos o más coches & 5,5 & 16,3 & 196 \\
Compact disc & 1,5 & 40,9 & 2.627 \\
Microondas & 1,6 & 39,2 & 2.350 \\
Secadora ropa & 3,6 & 7,7 & 114 \\
Lavavajillas & 5,4 & 16,2 & 200 \\
Aspiradora & 19,9 & 40,6 & 104 \\
\hline
\end{tabular}

$1[(1997 * 100) / 1998]-100$.

de energía que se vino produciendo a lo largo de algunas décadas. Se ha mencionado que aunque parece que la epidemia en Estados Unidos tiene su pico más definido a partir de la década de los ochenta, la obesidad y el sobrepeso no son un fenómeno nuevo, y ya en los años 60 los porcentajes de la población americana con un IMC $\geq 25 \mathrm{~kg} / \mathrm{m}^{2}$ eran del 48 y el $39 \%$ en hombres y mujeres respectivamente (Flegal, 1999). Este argumento, que hablaría de cierto efecto cohorte de la influencia de la ingesta energética en la prevalencia de obesidad, queda sin embargo cuestionado por la generalización de la epidemia a todos los grupos sociodemográficos incluyendo los niños y los adolescentes, en los que las prevalencias de sobrepeso y obesidad están aumentando (Hill \& Peters, 1998).

El otro conjunto de factores que se relacionan con la epidemia de obesidad son aquellos relativos al gasto de energía. El gasto de energía en su conjunto no se puede medir más que en condiciones controladas. Por ello, en Salud Pública sólo puede accederse, con enormes dificultades dada su complejidad, a la actividad física voluntaria. Todo parece indicar que las sociedades actuales, debido a la enorme mecanización y tecnificación de muchas de las tareas que realizan los individuos, son sedentarias. Diversos indicadores reflejados en el presente trabajo muestran la situación en España, donde se ha incrementado tanto el número de vehículos por habitante como el número medio de minutos de visionado de televisión, tendencia similar a la de otros países (French et al., 2001; Prentice \& J ebb, 1995). Al lado de estos indicadores de uso común, se suele argumentar que la enorme transformación tecnológica en los lugares de trabajo y en el hogar es- 
taría produciendo un descenso en el gasto de energía. Respecto al equipamiento, como muestran los datos presentados, se ha producido un importante incremento en el porcentaje de hogares que disponen de aparatos de televisión, vídeo, ordenador y otros elementos que han contribuido a un descenso en la energía necesaria para llevar a cabo ciertas tareas. Los datos presentados muestran el importante incremento en el número de hogares con dos o más aparatos de televisión (desde un 22\% en 1988 hasta un 58\% en 1997), incremento igualmente observado en Estados Unidos, donde estos porcentajes pasaron del 50\% en 1980 al $76 \%$ en el año 2000 (French et al., 2001).

En relación con la actividad física en el trabajo, el gasto de energía se ha reducido. En España, el porcentaje de trabajadores sedentarios aumentó entre 1987 y 1997 (Regidor \& Gutiérrez-Fisac, 1999). En otros países es evidente la reducción del gasto energético debido a la actividad laboral (Fogelholm et al., 1996; French et al., 2001). Sin embargo, la asociación entre la actividad física desarrollada durante el tiempo de trabajo y la obesidad es inconsistente, habiendo numerosos trabajos de investigación que no encuentran una relación entre ambas circunstancias (French et al. , 1994; GutiérrezFisac et al., 2002; J effery et al., 1991). Posiblemente es la actividad física en el tiempo libre y durante los desplazamientos al trabajo, más que la actividad propiamente laboral, la que están influyendo en el incremento de la prevalencia de obesidad.

La mayoría de estos indicadores indirectos de sedentarismo, y su tendencia creciente, hacen hincapié en la importancia del gasto energético en la actual epidemia de obesidad. Sin embargo, la actividad física en el tiempo libre declarada por los individuos no ha disminuido, sino que ha aumentado durante los últimos años. Los datos presentados muestran que el porcentaje de población que no realiza ningún ejercicio físico durante su tiempo libre ha descendido en España entre la década de los años ochenta y la de los noventa. Esta reducción es, además, importante, pasando el porcentaje de población sedentaria de un 54 y un $71 \%$ en 1987 a un 39 y un $52 \%$ en 1997 en hombres y mujeres respectivamente. Esta misma tendencia descendente del porcentaje de población sedentaria en el tiempo libre se ha visto en otros países en los que la prevalencia de obesidad está aumentando. Así, en Estados Unidos se comprueba igualmente un ligero descenso del porcentaje de población que declara no realizar ninguna actividad física en su tiempo libre, si bien la tendencia está estabilizada en los últimos años (CDC, 2000). Un interesante trabajo realizado en Finlandia (Fogelholm et al., 1996) puso de manifiesto el incremento en el número medio de kilocalorías consumidas entre 1982 y 1992 debidas al ejercicio en el tiempo libre. Junto a ello, es evidente también un aumento en la disponibilidad de instalaciones deportivas y lugares de recreo donde realizar ejercicio físico (French et al., 2001; INE, 1994a).

De esta manera nos encontramos ante una situación paradójica: a pesar del mayor uso de instrumentos (de ocio, de transporte) que implican una actitud sedentaria, el porcentaje de población que se declara activa en su tiempo libre es cada vez mayor. Para explicar esta paradoja podríamos argumentar que los instrumentos que suelen recoger la información sobre actividad física en tiempo libre son incompletos, y que reflejan sólo una parte (generalmente la relacionada con la práctica deportiva) de la actividad desarrollada durante el tiempo libre. Si bien las limitaciones de los instrumentos de medida de la actividad física de las poblaciones son evidentes, la tendencia de los indicadores indirectos presentados (número de vehículos, uso de la televisión, equipamientos de los hogares) es suficientemente sólida y consistente como para ser tenida en cuenta. Por ello, es también posible que la explicación de esta aparente paradoja esté en el hecho de que el tiempo libre disponible haya aumentado. Si lo que podríamos denominar "stock de tiempo libre" está aumentando en nuestras sociedades, un mayor porcentaje de población que realiza alguna actividad física en su tiempo libre y, a la vez, un mayor comportamiento sedentario de la población podrían ser circunstancias compatibles. Ese tiempo "extra" de ocio es el que estaría ocupado mayoritariamente por actividades sedentarias relacionadas con la televisión y el resto de medios de comunicación, cuya disponibilidad y uso están aumentando extraordinariamente en nuestras sociedades.

Los datos mostrados, consistentes con los de otros países, sugieren que los factores relacionados con la disminución del gasto de energía tendrían tanta o más importancia que los relativos a la ingesta en la tendencia creciente de la prevalencia de obesidad. Sin embargo, no podemos identificar claramente los cambios ni en los patrones de comportamiento alimentario ni en la actividad física de las poblaciones que expliquen el dramático aumento de la prevalencia del sobrepeso y la obesidad que se ha producido. La necesidad de obtener una información mejor sobre estos aspectos es evidente.

Lo que parece más claro es que los factores responsables tienen un denominador común: 
afectan de forma indiscriminada a la mayoría de los individuos de muy distintas sociedades. Son factores íntimamente relacionados con el desarrollo socioeconómico y, como ha sugerido Flegal (1999), más que un aumento en el número de casos de obesidad (que definiría epidemiológicamente una epidemia) estaríamos ante un enorme desplazamiento de toda la distribución del IMC de la población, hecho que señalaría el carácter de fenómeno de masas del sobrepeso y la obesidad y la profunda penetración social de unos factores que están íntimamente ligados al desarrollo socioeconómico de las sociedades actuales.

\section{Referencias}

AIMC (Asociación para la Investigación de Medios de Comunicación), 2001. Marco General delos Medios en España 2001. 23 Enero 2002 বhttp:// www. aimc.es/ $>$.

BINKLEY, J. K.; EALES, J. \& JEKAN OWSKI, M., 2000. The relation between dietary change and rising US obesity. International Journal of Obesity, 24:1032-1039.

BJÖRNTORP, P., 1997. Obesity. Lancet, 350:423-426.

CDC (Centers for Disease Control and Prevention), 2000. Behavioral Risk Factor Surveillance System. Trend Data. 26 February 2002 বhttp:// www.cdc. gov/nccdphp/brfss/ $>$.

DEPARTMENT OF HEALTH, 1995. The Health of the Nation. Fit for the Future. Second Progress Report on the Health of the Nation. London: Department of Health.

FLEGAL, K. M., 1999. The obesity epidemic in children and adults: Current evidence and research issues. Medicine and Science in Sports and Exercise, 31(Sup.):S509-S514.

FLEGAL, K. M.; HARLAN, W. R. \& LANDIS, J. R., 1988a. Secular trends in body mass index and skinfold thickness with socioeconomic factors in young adult women. American Journal of Clinical Nutrition, 48:535-543.

FLEGAL, K. M.; HARLAN, W. R. \& LANDIS, J. R., 1988b. Secular trends in body mass index and skinfold thickness with socioeconomic factors in young adult men. American Journal of Clinical Nutrition, 48:544-551.
FOGELHOLM, M.; MÄNNISTÖ, S.; VARTIAINEN, E. \& PIETINEN, P., 1996. Determinants of energy balance and overweight in Finland 1982 and 1992. International Journal of Obesity, 20:1097-1104.

FRENCH, S. A.; HARNACK, L. \& JEFFERY, R. W., 2000. Fast food restaurant use among women in the Pound of Prevention study: Dietary, behavioral and demographic correlates. International Journal of Obesity, 24:1353-1359.

FRENCH, S. A.; JEFFERY, R. W.; FOSTER, J. L.; MCGOVERN, P. G.; KELDER S. H. \& BAXTER, J. E., 1994. Predictors of weight change over two years among a population of working adults: The Healthy Worker Project. International Journal of Obesity, 18:145-154.

FRENCH, S. A.; STORY, M. \& JEFFERY, R. W., 2001. Environmental influences on eating and physical activity. Annual Review of Public Health, 22:309335.

GALUSKA, D. A.; SERDULA, M.; PAMUK, E.; SIEGEL, P. Z. \& BYERS, T., 1996. Trends in overweight among US adults from 1987 to 1993: A multistage telephone survey. American Journal of Public Health, 86:1729-1735.

GUTIERREZ-FISAC, J. L.; BANEGAS-BANEGAS, J. R.; RODRIGUEZ-ARTALEJO, F. \& REGIDOR, E., 2000. Increasing prevalence of overweight and obesity among Spanish adults, 1987-1997. International Journal of Obesity, 24:1677-1682.

GUTIERREZ-FISAC, J. L.; GUALLAR-CASTILLON, P.; DIEZ-GAÑAN, L.; LOPEZ-GARCIA, E.; BANEGAS- 
BANEGAS, J. R. \& RODRIGUEZ-ARTALEJO, F., 2002. Work-related physical activity is not associated with body mass index and obesity. Obesity Research, 10:270-276.

HEINI, A. F. \& WEINSIER, R. L., 1997. Divergent trends in obesity and fat intake patterns: The American paradox. American Journal of Medicine, 102:259264.

HILL, J. O. \& MELANSON, E. L., 1999. Overview of the determinants of overweight and obesity: Current evidence and research issues. Medicine and Science in Sports and Exercise, 31(Sup.):S515-S521.

HILL, J. O. \& PETERS, J. C., 1998. Environmental contributions to the obesity epidemic. Science, 280: 1371-1374.

HOFFMEISTER, H.; MENSINK, G. B. M. \& STOLZENBERG, H., 1994. National trends in risk factors for cardiovascular disease in Germany. Preventive Medicine, 23:197-205.

INE (Instituto Nacional de Estadística), 1994a. Anuario Estadístico 1993. Madrid: INE.

INE (Instituto Nacional de Estadística), 1994b. Encuesta de Presupuestos Familiares 90-91. Volumen 2. Consumo de Alimentos, Bebidas y Tabaco en Unidades Físicas. Madrid: INE.

INE (Instituto Nacional de Estadística), 2001. Anuario Estadístico de España 2000. Madrid: INE.

JEFFERY, R. W. \& FRENCH, S. A., 1998. Epidemic of obesity in the United States: Are fast food and television viewing contributing? American Journal of Public Health, 88:277-280.

JEFFERY, R. W.; FRENCH, S. A.; FOSTER, J. L. \& SPRY, V. M., 1991. Socioeconomic status differences in health behaviors related to obesity: The Healthy Worker Project. International Journal of Obesity, 15:689-696.

KHAN, L. K. \& BOWMAN, B. A., 1999. Obesity: A mayor global public health problem. Annual Reviews of Nutrition, 19:18-19.

KUSKOWSKA-WOLK, A. \& BERGSTRÖM, R., 1993a. Trends in body mass index and prevalence of obesity in Swedish men 1980-89. Journal of Epidemiology and Community Health, 47:103-108.

KUSKOWSKA-WOLK, A. \& BERGSTRÖM, R., 1993b. Trends in body mass index and prevalence of obesity in Swedish women 1980-89. Journal of Epidemiology and Community Health, 47:195-199.

MAILLARD, G.; CHARLES, M. A.; THIBULT, N.; FORHAN, A.; SERMET, C., BASDEVANT, A. \& ESCHWĖGE, E., 1999. Trends in the prevalence of obesity in the French adult population between 1980 and 1991. International Journal of Obesity, 23:389-394.
MCCRORY, M. A.; FUSS, P. J.; HAYS, N. P.; VINKEN, A. G.; GREENBERG, A. S. \& ROBERTS, S. B., 1999. Overeating in America: Association between restaurant food consumption and body fatness in healthy adult men and women ages 19 to 80 . Obesity Research, 7:564-571.

NIETO-GARCIA, F. J.; BUSH, T. L. \& KELY, P. M., 1990. Body mass index definition of obesity: Sensitivity and specificity using self-reported weight and height. Epidemiology, 1:146-152.

NIH (National Institutes of Health), 1998. Clinical Guidelines on the Identification, Evaluation and Treatment of Overweight and Obesity in Adults. The Evidence Report. 20 February 2001 «ttp:// www.nhlbi.nih.gov/guidelns/obesity/ob_gdln>.

PI-SUNYER, F. X., 1994. The fattening of America. JAM A, 272:238-239.

POPKIN, B. M. \& DOAK, C. M., 1998. The obesity epidemic is a worldwide phenomenon. Nutrition Reviews, 56:106-114.

PRENTICE, A. M. \& JEBB, S. A., 1995. Obesity in Britain: Gluttony or sloth. BMJ, 311:437-439.

REGIDOR, E. \& GUTIERREZ-FISAC, J. L., 1999. Indicadores de Salud. Cuarta Evaluación en España del Programa Regional Europeo Salud para Todos. Madrid: Ministerio de Sanidad y Consumo.

RODRIGUEZ-ARTALEJO, F.; LOPEZ-GARCIA, E.; GUTIERREZ-FISAC, J. L.; BANEGAS-BANEGAS, J. R.; LAFUENTE-URDINGIO, P. J.; DOMINGUEZ-ROJAS, V., 2002. Changes in the prevalence of overweight and obesity and their risk factors in Spain, 1987-1997. Preventive Medicine, 34:72-81.

RODRIGUEZ-ARTALEJO, F.; BANEGAS, J. R.; GRACIANI, M. A.; HERNANDEZ-VECINO, R. \& REYCALERO, J., 1996. El consumo de alimentos y nutrientes en España en el periodo 1940-1988. Análisis de su consistencia con la dieta Mediterránea. Medicina Clínica (Barcelona), 106:161-168.

SEIDELL, J. C., 1995. Obesity in Europe: Scaling an epidemic. International Journal of Obesity, 19 (Sup. 3):S1-S4.

SEIDELL, J. C.; VERSCHUREN, W. M. M. \& KROM HOUT, D., 1995. Prevalence and trends of obesity in the Netherlands 1987-1991. International Journal of Obesity, 19:924-927.

STAM LER, J., 1993. Epidemic of obesity in the United States. Archives of Internal Medicine, 153:10401044.

UAUY, R.; ALBALA, C. \& KAIN, J., 2001. Obesity trends in Latin America: Transiting from under to overweight. Journal of Nutrition, 131:899S-899S.

WHO (World Health Organization), 2000. European Health for all Database. 1 June 2001 ४ttp:// www. euro.who.int/hfa>

Recibido el 22 de abril de 2002

Versión final presentada el 12 de agosto de 2002

Aprobado el 24 de septiembre de 2002 